\title{
New York dans le polar métaphysique : la ville totale
}

\author{
Delphine Carron
}

\section{(2) OpenEdition}

Journals

\section{Édition électronique}

URL : http://journals.openedition.org/transtexts/134

DOI : 10.4000/transtexts.134

ISSN : 2105-2549

\section{Éditeur}

Gregory B. Lee

\section{Édition imprimée}

Date de publication : 1 septembre 2007

Pagination : 26-42

ISSN : 1771-2084

\section{Référence électronique}

Delphine Carron, « New York dans le polar métaphysique : la ville totale», Transtext(e)s Transcultures 跨 文本跨文化 [En ligne], 3 | 2007, mis en ligne le 15 octobre 2009, consulté le 20 avril 2019. URL : http:// journals.openedition.org/transtexts/134; DOI : 10.4000/transtexts.134 


\title{
New York dans le polar métaphysique : la ville totale
}

\author{
DeLPHINE CARRON
}

Thousands of writers have set their novels in New York City and several anthologies of New York writings have been published recently, establishing the city as a central element in American literature. Considering the particular genre of detective fiction, this is also the case, so to speak. Our contemporary world, mostly urban, has led to a major shift in the genre towards increasing uncertainty and doubt about the traditional detective hero's ability to cope with such a harsh environment. He used to stand as a reassuring heroic figure against the historic background inherited after WWII and then the Cold War and the Vietnam War. Then followed an era of increasing terrorism, globalization and generalized scare; September 11 came as the ultimate manifestation of this threat. In this context, detective fiction mostly evolved towards a much darker path - thriller or noir - whereas other authors created a new branch of the genre by mixing detective fiction patterns with a metaphysical questioning of our contemporary existence - metaphysical detective fiction - which can be illustrated by writers from very different horizons, such as Paul Auster in his New York Trilogy, Jerome Charyn and his Isaac Sidel series or Jonathan Lethem's Motherless Brooklyn. All three have in common their renewal of the detective genre and their deep attachment to New York. The city is the main protagonist of their detective fiction: omnipresent and omnipotent, New York plays a capital role in the narration and stands as a genuine participant in the plot.

New York City est le cadre choisi par de nombreux auteurs, américains ou non. Plusieurs anthologies consacrent de même la ville comme un élément central du 
champ littéraire américain, et de la littérature en général. Si l'on considère plus particulièrement le genre du polar, on constate que la ville y joue là aussi un rôle prépondérant. Nos sociétés contemporaines, majoritairement urbaines, ont amené ce genre à de profondes mutations vers un univers de plus en plus incertain, où le doute s'est installé quant à l'aptitude du détective à évoluer et à conclure son enquête dans un cadre aussi éprouvant. Il remplissait jusqu'alors le rôle de héros rassurant face à un univers urbain rendu de plus en plus hostile après les stigmates laissés par la seconde guerre mondiale, puis la guerre froide et le conflit au Vietnam. Ces évènements ont ouvert une période caractérisée par la mondialisation, la montée du terrorisme et une peur fondamentale encore attisée par le traumatisme du 11 septembre 2001.

Dans ce contexte, le polar américain évolue globalement vers plus de noirceur à travers le 'thriller' ou le 'noir'. Dans le même temps, une nouvelle voie s'est créée au sein du polar en alliant les motifs du polar à un questionnement métaphysique et introspectif sur la société contemporaine et la place de l'individu au cœur de celle-ci. Cette branche nouvelle prend le nom de polar métaphysique ou 'metaphysical detective fiction' et réunit des auteurs très différents, tels les trois retenus ici : Paul Auster, auteur de The New York Trilogy et de Squeeze Play, un polar paru sous le pseudonyme de Paul Benjamin, Jerome Charyn et sa série de polars consacrée au détective récurrent Isaac Sidel, et Jonathan Lethem avec les romans Motherless Brooklyn et Gun, with Occasional Music. Ces trois auteurs partagent la même volonté d'apporter un renouveau au polar - notamment par le biais de la bande dessinée - et le même attachement à New York. La ville est en effet le personnage principal de leurs récits ; omniprésente et toute-puissante, New York joue un rôle clé dans la redéfinition du polar et incarne la figure urbaine par excellence.

On trouve au fil des polars métaphysiques de Paul Auster, Jerome Charyn et Jonathan Lethem de nombreuses occurrences de l'expression «This is New York». Nul besoin d'en dire plus, cette simple phrase clôt tout débat sur la possibilité d'appréhender la ville, de la comprendre : rien ne sert de chercher à trouver un sens à ce qui se passe à New York, les choses sont ainsi parce qu'il s'agit de New York, ville insaisissable qui résiste à toute tentative de normalisation. Jerome Charyn la présente ainsi dans une discussion entre Isaac Sidel, candidat réticent à la mairie de New York, Rebecca Karp, l'actuelle maire, et Mario, son secrétaire particulier qui pousse Isaac à se présenter :

"And all you'll ask is that I save a room for Rebecca at the mansion." 
“That's about it. Rebecca would rather have her Isaac than some pol who's been stabbing her in the back ... the town loves you. You'll have to get used to that."

"But will it love me when I can't deliver?"

Rebecca started to crackle in her rocking chair. "I told you he's a klutz ... Isaac, this is New York. Nobody can deliver."1

Les mots de Rebecca sont lourds de sous-entendu: le maire comme tout autre habitant reste soumis à la ville. Cette fatalité urbaine détermine l'existence de chacun de ses habitants et du détective en particulier qui, dans sa volonté d'aller au devant de la vérité, se trouve seul face à l'implacable puissance de la ville.

Celle-ci prend alors des allures de véritable personnage, à la fois personnifiée et déshumanisante, qui semble drainer ses habitants de leurs attributs humains pour les accaparer et les faire siens, comme le souligne Jean-Noël Blanc dans Polarville :

«Ville impitoyable. Ville enfin devenue personnage: hostile, menaçante, c'est elle qui engendre la peur, comme un être doté de personnalité. ... C'est donc accorder autant d'importance aux tirades consacrées à la ville qu'aux faits et gestes des autres acteurs.»²

Cette représentation symbolique de New York tend vers une figure mythique et impitoyable, souvent féminine, qui incarne une menace et non plus un havre pour l'individu. On trouve une telle représentation de la ville à l'ouverture du roman graphique de Jerome Charyn Margot Queen of the Night qui montre une Margot transformée par son arrivée à New York et la vie extrêmement dure qu'elle y mène ensuite: ${ }^{3}$

Endurcie, devenue impitoyable pour ses ennemis, Margot a quitté l'apparence de la jeune fille provinciale pour devenir une femme urbaine qui a construit son image extérieure telle une carapace de protection contre la dureté de la ville, qu'elle finit par incarner. Le dessin en légère contre-plongée, le regard dur et les cheveux courts transforment Margot en meneuse d'équipe implacable dont la mission est de détruire. Celle-ci lui est imposée par la ville, dont les lois s'apparentent à celles d'une véritable jungle urbaine : si Margot ne domine pas, alors elle sera dominée.

'Jerome Charyn, Montezuma's Man, New York, Mysterious Press, 1993, p.130.

2Jean Noël Blanc, Polarville - Images de la ville dans le roman policier, Lyon, Presses Universitaires, 1991, pp. $42-43$

3Jerome Charyn and Massimo Frezzato, Margot Queen of the Night, New York, Heavy Metal, 1995, p.1. 
Dans le cadre du polar métaphysique et de sa distorsion des éléments du polar traditionnel, New York s'apparente alors au personnage conventionnel de la femme fatale, à laquelle on ne peut résister mais qui se révèle être un piège inéluctable. Jean-Noël Blanc intitule d'ailleurs un passage de Polarville «Ville perverse, femme fatale». Le détective de polar métaphysique a dû apprendre à se méfier, aussi lorsque dans Gun, with Occasional Music le détective Conrad Metcalf décrit la ville comme une femme fatale, il se doute du piège qu'elle lui tend sans pour autant l'éviter: «I was stupid enough to think there was something wrong with the silence that had fallen like a gloved hand onto the bare throat of the city».4 Une nouvelle fois, la ville se fait l'écho des personnages: Metcalf, confronté aux avances douteuses de l'attirante Celeste Stanhunt, accède en se tournant vers la vue de la ville par la fenêtre à une prise de conscience du danger que représente Celeste et parvient à la repousser.

Toutefois le polar va plus loin que la représentation de la ville comme force castratrice opposée au détective. En effet, la ville est le plus souvent asexuée, et surtout inhumaine. Aliénante et dominatrice, elle prend des allures de monstre urbain et ses éléments caractéristiques, les gratte-ciels, prennent vie. A l'inverse, la vie de ses habitants se fige et se minéralise. L'adaptation en bande dessinée Paul Auster's City of Glass illustre cette double tendance en représentant Quinn littéralement avalé par le mur de briques tandis que le gratte-ciel prend vie sous forme de monstre de pierre: 5

Jerome Charyn reprend aussi cette image dans son essai sur New York, Metropolis, et cite Lewis Mumford: «New York had little more to give than «shapeless giantism» and «megalopolitan growth». It was a «Plutonian world, in which living forms became frozen into metal». Mumford offered us an entire schema, «A Brief Outline of Hell». ${ }^{6}$ A l'origine une entreprise humaine, l'espace urbain a pris le dessus et inversé les rôles. New York est alors désignée par Jonathan Lethem par l'expression «Manhattan's dinosaur spine» ou bien «the whole watery mouth of the city» et évoque ainsi un véritable monstre urbain, à la fois gigantesque et effrayant. ${ }^{7}$ Jerome Charyn reste le plus prolixe en matière de représentation monstrueuse de New York; dans Metropolis il définit la ville comme «...a python lurking around. ... New York was the dream that dreamt itself, a monster out of the Old World that

\footnotetext{
"Jonathan Letham, Gun, with Occasional Music, New York: Tor Books, 1994, p.130.

${ }_{5}^{5}$ Paul Auster, Paul Karsik, David Mazzucchelli, and Art Spiegelman, Paul Auster's City of Glass, New York, Avon Books, 1994, p.101.

6Jerome Charyn, Metropolis: New York as Myth, Marketplace, and Magical Land, New York, Putnam, 1986, p.39.

${ }^{7}$ Jonathan Letham, The Fortress of Solitude, New York, Doubleday, 2003, p.79 et p.239.
} 
could never rest, spitting ideals like fireworks - liberty, brotherhood, and zerocoupon bonds». ${ }^{8}$ La monstruosité de la ville est surtout évoquée par ses gratte-ciels, que Charyn qualifie de «brick teeth», et par ses structures les plus imposantes, en particuliers le Brooklyn Bridge: «Isaac ... walked off the Brooklyn Bridge, into the belly of Manhattan. ... The bottom of the Brooklyn Bridge reminded Isaac of the flattened belly of a monster snail». ${ }^{9}$ Les habitants constituent la matière première qui la nourrit: insignifiante, leur foule se reconstitue sans discontinuer par les incessants apports de l'immigration: «Like the Irish before us, and the Italians, we scraped our character upon the surface of the City, and inside its bowels». ${ }^{10}$ Jonathan Lethem reprend cette image dépréciée de New York comme 'melting pot' dans l'entretien qu'il m'a accordé en novembre 2004:

«The immigrant civilization remains in a permanent state of delirious confusion in New York. The city is the place where you come to transform yourself into an American. If you succeed, you move away into the suburbs, or to some other state. If you fail, you become a New Yorker - often a Brooklynite, instead. The city is addicted to the energy and strife of discordant populations attempting to negotiate a peaceful existence side-by-side in close proximity. ... At the same time, the result is deeply sinister, futuristic, and dystopian. New York is a city of alienation and fear - and if you underestimate this, you'll fail to survive. ${ }^{11}$

La ville d'ordinaire définie comme lieu d'intégration est ici vue comme un monstre engloutissant l'immense variété des populations qui la composent pour en produire des individus à son image qui ne quitteront plus la ville. Car si New York n'étanche jamais son besoin d'absorber l'énergie de ses habitants, ces derniers, une fois fondus dans le moule new-yorkais, dépendent trop d'elle pour la quitter. L'addiction est donc à double tranchant. Isaac Sidel lui-même ne peut vivre hors de New York: «What could I do in Seattle? I'd miss the cockroaches. I'd go woolly without the street». ${ }^{12}$ Cette addiction est même associée à la folie chez Jerome Charyn, à la fois «schizophrenia» et «lunacy»:13

«Inside that winnowing town, a murderous city that ate its population to bits, we possessed those lunatic ideals, a laundryman's faith in the immigrant ... that the murderous city was also a city of marvels. ... New York loved and

8Jerome Charyn, Metropolis: New York as Myth, Marketplace, and Magical Land, p.132 and p.217.

${ }^{9}$ Charyn, Metropolis, p.234.

Jerome Charyn, Gun, with Occasional Music, p.93 and p.101.

${ }^{10}$ Jerome Charyn, Metropolis: New York as Myth, Marketplace, and Magical Land, p.267.

${ }^{11 J o n a t h a n ~ L e t h e m, ~ « A c c e s s ~ F a n t a s y », ~ i n ~ M e n ~ a n d ~ C a r t o o n s, ~ N e w ~ Y o r k: ~ D o u b l e d a y, ~} 2004$.

12Jerome Charyn, The Isaac Quartet (1974-1978), New York: Four Walls Eight Windows, 2002, p.306.

13Jerome Charyn, Metropolis: New York as Myth, Marketplace, and Magical Land, p.23 and p.209. 
killed, both were part of its fabric and constitution, its uniqueness among all other cities, because no other city in the world had ever defined itself by the numbers of immigrants it could raise as its very own children. ... The city is their companion and their mother, their schoolmaster and executioner. $»^{14}$

Les plus grandes puissances se voient dominées par la loi implacable de la ville, comme le rappelle Woody Haut dans Neon Noir: «In the end, Isaac knows that no one can control the city. Says Charyn, It's too powerful a beast ... It doesn't matter if it's the Mafia or the FBI, they're all eaten up by the city». ${ }^{15}$ Jerome Charyn replace alors New York dans la thématique mythique et la compare tout au long de Little Angel Street au Léviathan, tout comme d'ailleurs Paul Auster dans le roman du même nom. Monstre aquatique à plusieurs têtes dans la Bible, le livre d'Enoch le présente comme une créature monstrueuse féminine régnant sur les abysses et le monde aquatique en général. Reprise par Thomas Hobbes au XVII ème siècle, l'allégorie du Léviathan désigne cette fois le pouvoir absolu d'une entité souveraine établie par un contrat social entre les hommes pour faire cesser la discorde.

Dans le polar contemporain, c'est la ville qui incarne cette entité monstrueuse et autoritaire contre laquelle les habitants ne peuvent se retourner pour les raisons suivantes : ils ont choisi et créé la ville en premier lieu et surtout, la ville les a isolés les uns des autres. Fondamentalement seuls, ils luttent sans espoir de parvenir à se détacher de l'emprise de la ville: «We're living inside a Leviathan. I can't change things» constate Isaac Sidel. ${ }^{16}$ Le monstre ronge la structure même de New York, «the City [that] never sleeps». ${ }^{17} \mathrm{~L}$ 'autorité municipale reste impuissante face à la dureté de la ville et doit trouver l'argent qui lui fait cruellement défaut en sacrifiant ses propres employés:

«Nicholas attacked the Leviathan of New York City, chopped off whole departments, shuffled men and women around. But Isaac would start to dream whenever he had to examine the City's books. He wasn't like Nicholas. He couldn't fight the Leviathan, claw by claw. The king had too many details inside his skull. ... There would be a shortfall in fiscal eighty-six unless Nicholas continued to chop and chop. ${ }^{18}$

L'utopie humaine de la grande ville vitrine de la puissance de l'homme n'est plus de mise. On assiste même à des ébauches de dystopies urbaines, tel cet exemple tiré

\footnotetext{
${ }^{14}$ Charyn, Metropolis, p.252.

${ }^{15}$ Woody Haut, Neon Noir: Contemporary American Crime Fiction, London, Serpent's Tail, 1999, p.199.

16Jerome Charyn, Little Angel Street, New York, Mysterious Press, 1994, p.8.

${ }^{17}$ Jerome Charyn, El Bronx, New York, Mysterious Press, 1997, p158.

${ }^{18 J e r o m e ~ C h a r y n, ~ L i t t l e ~ A n g e l ~ S t r e e t, ~ p p .209-10 ~}$
} 
du Geek de New York de Jerome Charyn : «Elle voyait des voiturettes. Des milliers. Une centaine de milliers de wagons de glaces. Qui dirigeaient la ville. Qui avaient placé Manhattan sous la loi martiale. Qui vous renversaient si vous tentiez de traverser la rue»..$^{19}$ Le rythme fragmenté évoque la frénésie qui s'est emparée de l'univers urbain; l'œuvre s'est retournée contre son créateur à une époque où l'homme lui-même s'est majoritairement détourné de tout dieu. La ville défiante des puissances supérieures, la Babel moderne, se change en construction menaçante, écrasante. New York présente alors son pire visage : Gotham, «the ultimate triumph of the grid».20

Gotham, terme utilisé pour la première fois par Washington Irving en 1807 pour désigner New York, fait référence à une légende anglaise du XIII ème siècle selon laquelle les habitants du village de Gotham, mécontents d'apprendre que le roi souhaitait acheter une de leurs terres, se seraient comportés en fous lors de sa visite au village pour l'en dissuader. L'allusion d'Irving est donc ironique : New York s'apparente à un lieu où le désordre règne à double titre - folie et insubordination - et demeure donc impossible à cerner et à maîtriser. Gotham reste de nos jours surtout associée à la violence et l'insécurité mises en avant dans les nombreux épisodes de Batman. Cette image d'une ville peu sûre, terrifiante, est rendue par la démesure des dimensions urbaines, l'infinité horizontale et surtout l'explosion verticale que l'on trouve dans toute la bande dessinée urbaine contemporaine : «Exagération des perspectives, torsion des plans, déformation des lignes de fuite, désarticulation des angles de vue, brisures systématiques des droites et autres artifices composent un espace véhément et discordant qui exprime l'inquiétude d'un univers oppressant». ${ }^{21}$ Les polars graphiques de Jerome Charyn répondent à cette tendance et y ajoutent d'autres procédés de représentation de la ville sous un angle qui écrase et menace l'individu. Ainsi dans Margot in Badtown, le sommet des gratte-ciels inhumains et froids dépasse-t-il les limites de la case tandis que The Magician's Wife présente une vue plongeante de Manhattan où les angles de vues se trouvent légèrement déformés pour procurer une sensation de puissance et de domination:22

\footnotetext{
${ }^{19}$ Jerome Charyn et Michel Martens, Geek de New York, Paris, Gallimard, 1995, p.139. ${ }^{20}$ Jerome Charyn, Metropolis: New York as Myth, Marketplace, and Magical Land, p.236. ${ }^{21}$ Jean Noël Blanc, Polarville - Images de la ville dans le roman policier, p.50.

${ }^{22}$ Jerome Charyn et Francois Boucq, The Magician's Wife, New York, Catalan Communications, 1987, p.39; Jerome Charyn et Massimo Frezzato, Margot Queen of the Night, New York, Heavy Metal, 1995, p.12.
} 
Plus loin, les arbres de Central Park prennent des allures de piliers d'église ; la nuit et la solitude du personnage dans un tel lieu renforcent l'aspect terrifiant de cette case. $^{23}$

Le protagoniste se trouve en effet toujours menacé, en passe d'être englouti ou mis à mal par l'univers urbain. New York enserre donc ses habitants, les étouffe. Cette représentation littéraire de la ville ne semble pas si éloignée de la réalité à en juger par les propos de Paul Auster dans La Solitude du labyrinthe, lui-même soumis à une influence ambiguë de la part de la ville :

«Je ne sais pas pourquoi mais tous les gens que je connais se plaignent de cela. Tout le monde est très occupé. La vie quotidienne à New York est dure. Et cela prend beaucoup de temps simplement pour exister. ... J'aime énormément New York. C'est une source d'inspiration et de pensées. Aucune autre ville ne lui ressemble. Dans le même temps, je déteste cette ville si difficile, tout en reconnaissant que je dois sans doute avoir besoin de cette difficulté - un peu comme ce studio de travail difficile car inconfortable. New York est une ville inconfortable.» ${ }^{24}$

Auster est aussi l'auteur d'un guide intitulé The Gotham Handbook à l'attention de l'artiste Sophie Calle, preuve que la vie new-yorkaise nécessite une adaptation, une initiation. Dans cet ensemble de pierre, de verre et de métal, les perspectives sont réduites et l'atmosphère oppressante. Le poids de la réalité urbaine partout écrase, on ne semble pouvoir s'évader :

«La ville est là. Indiscutable. Donnée. Présente au même titre qu'une réalité minérale. C'est une réalité lourde. Massive. Ecrasante.

Pas d'échappée. Pas de ciel. Pas de vastes avenues débouchant sur un ailleurs un peu plus riant. Toutes les perspectives sont bouchées.»25

Autant le personnage ne parvient pas à se détacher de la ville, autant, lorsqu'il en est séparé, naît en lui un profond malaise ; il ne reconnaît plus l'environnement urbain, il ne se reconnaît plus lui-même. C'est le cas du narrateur de The Locked Room de Paul Auster :

«The sky was more present than in New York, its whims more fragile. I found myself drawn to it, and for the first day or two I watched it constantly sitting in my hotel room and studying the clouds, waiting for something to happen.... The Paris sky has its own laws, and they function independently of the city below. ... This was an old world city, and it had nothing to do with

23Jerome Charyn et Francois Boucq, The Magician's Wife, p.64.

${ }^{24}$ Paul Auster et Gerard de Cortanze, La Solitude du labyrinthe, Paris, Babel, 2004, p.195 and p.198.

${ }^{25} J$ ean Noël Blanc, Polarville - Images de la ville dans le roman policier, p.57. 
New York - with its slow skies and chaotic streets, its bland clouds and aggressive buildings. I had been displaced, and it made me suddenly unsure of myself. $»^{26}$

L'agressivité de la ville pèse sur ses habitants et exerce sa pression par l'entremise des gratte-ciels, véritables colosses dépourvus d'humanité érigés là en envahisseurs de l'espace urbain, et parmi eux le plus terrifiant, le World Trade Center, décrit par Jerome Charyn dans Metropolis: «...the World Trade Center : Rockefeller's Folly. No pair of twins had ever assaulted New York in such a manner. Ruinous monoliths, they were without romance or play. They seized the sky like some hangman's dream of the twentieth century». ${ }^{27}$ Face à ces instruments de coercition, il ne reste à l'individu que deux alternatives: se plier et perdre tout libre arbitre dans son existence au sein de la cité, ou bien la défier: «Aggressiveness is a way of life in New York. One has to be an outlaw to survive. We're all gangsters over here, anonymous in that dark outline of the City, no matter what hearths we have». ${ }^{28} \mathrm{Le}$ détective se positionne alors difficilement par rapport à cet état particulier de horsla-loi puisqu'il ne s'agit ici que de se placer à l'écart de la loi imposée par la ville, et non de la loi telle que l'entend le polar habituellement. Si l'on effectue un rapide survol, les détectives des polars de Paul Auster, Jerome Charyn et Jonathan Lethem vont au devant des lois implacables de la cité dans le cadre de leurs enquêtes mais finissent pour la plupart dans l'échec de leur mission et/ou dans un trouble intérieur aux séquelles irréversibles.

\section{La ville monochrome}

La représentation de la dureté de la ville offre alors, aussi bien dans les descriptions romanesques que graphiques, un espace urbain défini essentiellement par le noir et blanc et toute la déclinaison des gris : il n'y a presque plus de place pour la couleur. La ville monochrome reflète son implacabilité, l'impossibilité de s'en échapper. Elle durcit les angles, démultiplie les hauteurs et place le personnage tour à tour dans l'aveuglement de la lumière crue ou dans l'oubli des ombres obscures. La taille démesurée des immeubles masque le soleil, creuse d'immenses zones d'ombre et prive les habitants de la lumière vitale, comme le montre cet extrait de «Access Fantasy » de Jonathan Lethem: «a sliver of morning sun that shone across the middle of the block, benefit of a chink in the canyon of towers that surrounded

\footnotetext{
${ }^{26}$ Paul Auster, «The Locked Room», The New York Trilogy (1985-1986), New York, Penguin Books, 1990, p.338.

27Jerome Charyn, Metropolis: New York as Myth, Marketplace, and Magical Land, p.245.

${ }^{28}$ Jerome Charyn, Metropolis: New York as Myth, Marketplace, and Magical Land, p.269.
} 
them» ${ }^{29}$ Les gratte-ciels forment ainsi un paysage sauvage et abrupt tandis que leurs matériaux unis, durs et froids chassent la couleur de l'espace urbain: «La ville du polar répugne à la lumière franche. Sa palette est avare. Elle préfère les couleurs rares, pauvres, éteintes, lorsqu'elle ne va pas jusqu'à ne retenir que le noir et blanc et leur opposition violemment contrastée». ${ }^{30}$ C'est de cette manière que les concepteurs de Paul Auster's City of Glass ont présenté New York visuellement. La couleur en est absente et les dernières pages entièrement noires semblent happer le héros: ${ }^{31}$

La dichotomie entre noir et blanc souligne en effet le paradoxe du détective qui cherche à percer le mystère urbain, à faire la lumière sur ses secrets, mais qui, si la ville lui laisse entrevoir un de ses pans cachés, en reste aveuglé. Outre le duel entre obscurité et clarté, l'opposition noir/blanc évoque aussi la lutte entre le bien et le mal, l'innocence et la connaissance, et par extension la thématique religieuse.

\section{Utopies urbaines}

Dans une société contemporaine globalement post-religieuse, New York s'élève telle un défi aux dieux et aux hommes, et évoque une Babel moderne. Dans Squeeze Play la comparaison entre New York et Babel est d'ailleurs clairement établie, Max Klein passe de longs moments à contempler les neuf reproductions de la Toren Van Babel de Brueghel qu'il a accrochées dans son bureau:

«The picture shows the nearly finished tower reaching up towards the sky and scores of tiny workers and animals toiling away at the construction, diligently laboring over the most colossal monument to human presumption ever built. The painting never failed to make me think of New York, and it helped to remind me how our sweat and agony will always come to nothing in the end. It was my way of keeping things in perspective.» ${ }^{32}$

Cette volonté d'élévation démesurée se présente, à l'instar de la Tour de Babel, comme un nouveau défi aux dieux, un symbole d'affranchissement du pouvoir divin. En revanche, au contraire de l'épisode original, ce ne sont pas les habitants qui défient l'autorité implacable des dieux mais la ville, qui affirme son indépendance vis-à-vis des dieux mais aussi vis-à-vis des hommes : elle clame ainsi sa toute-puissance. Face à cette réalité qui ne laisse place à aucun autre choix, City of

29Jonathan Letham, «Access Fantasy», p.28.

${ }^{30}$ Jean Noël Blanc, Polarville - Images de la ville dans le roman policier, p.51.

${ }^{31}$ Paul Auster, Paul Karsik, David Mazzucchelli, et Art Spiegelman, Paul Auster's City of Glass, pp132-33.

${ }^{32}$ Paul Auster (sous le pseudonyme de Paul Benjamin), Squeeze Play (1982), New York, Penguin Books

Crime, 1990, p.18. 
Glass présente la renaissance de Babel comme paradis terrestre, l'espoir d'un retour au divin et à la concorde entre les langues et entre les hommes ainsi que l'expose Stillman dans son pamphlet :

«In the year 1960, he stated confidently, the new Babel would begin to go up, its very shape aspiring toward the heavens, a symbol of the resurrection of the human spirit. History would be written in reverse. What had fallen would be raised up; what had been broken would be made whole. Once completed, the Tower would be large enough to hold every inhabitant in the New World. There would be a room for each person, and once he entered that room, he would forget everything he knew. After forty days and forty nights, he would emerge a new man, speaking God's language, prepared to inhabit the second, everlasting paradise.»33

L'utopie de Stillman montre sa volonté de changer la ville telle qu'elle est : fragmentée, individualiste, incompréhensible, invivable. Il aspire à l'exact opposé : un espace de partage, de communauté. Cet idéal ressemble précisément à celui qui a, à l'origine, motivé la construction des grands centres urbains. Or ce retour à l'idée d'origine n'est plus possible et la théorie de Stillman ne se concrétise que dans sa folie. Elle appartient définitivement au domaine de l'utopie malgré les efforts de Stillman pour lui donner une apparence scientifique.

On trouve aussi un modèle de ville idéale dans le roman graphique de Jerome Charyn, Margot Queen of the Night. Il s'agit cette fois d'un projet immobilier, une enclave circulaire et protégée dans le labyrinthe rectiligne et délabré du nord de Manhattan: ${ }^{34}$

Ce prétendu paradis architectural n'est qu'un simulacre d'utopie: son immense enceinte de béton gris et sa taille gigantesque semblent écraser les quartiers voisins ; l'alternative à la ville implacable n'est pas viable, ce n'est qu'un refuge destiné aux plus riches, pour ne plus voir la ville dans sa dure réalité, mais elle ne permet pas de la quitter. Nul ne semble donc pouvoir s'évader de l'emprise urbaine ; la dureté de la réalité urbaine passe par le crime et l'absence de rédemption.

\section{Echapper à la ville}

Polarville dresse un bilan pessimiste de la ville qui définit tout particulièrement les polars de Paul Auster, Jerome Charyn et Jonathan Lethem : un espace qui influence

${ }_{33}^{3}$ Paul Auster, The New York Trilogy (1985-1986). New York, Penguin Books, 1990, p.59.

${ }^{34}$ Jerome Charyn et Massimo Frezzato, Margot Queen of the Night, p.29. 
l'individu, le pousse à fuir mais l'en empêche, lui donne l'illusion d'avancer - à travers l'enquête - mais suspend tout aboutissement :

«Or cette ville résume l'existence. Elle est le monde à elle seule. Rien ou presque ne se passe au dehors. Quelques rares évocations de la nature, peu de relations entre métropoles, presque pas de territoire national: l'espace du polar se borne à la ville. ... Nulle part c'est ici, pour toujours. Ailleurs n'existe qu'en rêve. Rêve impossible, rêve fou : la ville rêvée n'est pas la ville. Seule existe la ville réelle, d'où l'on ne s'échappe jamais véritablement, et où l'on meurt.»35

La dureté urbaine semble alors inéluctable et les alternatives peu nombreuses pour éviter la chute consécutive: chacun sombre dans la spirale de la perte. Le protagoniste y perd son identité, son foyer, ses relations, son argent et bien souvent on ne sait ce qu'il advient de lui ; la fin du roman nous ôte bien souvent tout espoir de dénouement. Jerome Charyn expose ainsi dans Family Man cette vision cauchemardesque de la ville, où le héros, Charles, est un prêtre qui travaille pour la police, parcourant les rues terrifiantes de New York en soutane et revolver. Son frère, Alonso, n'est autre que le dernier membre en fuite d'une famille mafieuse. Charyn présente la police comme seule entité salvatrice dans un espace où la pauvreté a poussé la plupart au crime. L'autorité et la justice divines se voient supplantées par une force policière expéditive et corrompue. Family Man ne présente donc pas d'échappée à la ville invivable, même le sacré se trouve sali car mêlé à la dureté de la ville. Toute idée de justice se trouve balayée par une police qui intervient masquée et violente les plus faibles ; c'est paradoxalement Alonso, le frère maudit de Charles, qui met en péril son anonymat pour prendre leur défense et faire régner un semblant de justice.

\section{Constats d'échec}

Les romans de Jerome Charyn reflètent aussi ce paradoxe : ce sont les malfrats qui perpétuent les usages de l'honneur et de la solidarité, ne laissant jamais un des leurs dans le besoin; les puissants ne font rien pour sauver la ville, ou bien s'avouent vaincus. C'est le cas en particulier pour Isaac Sidel, d'abord 'Police Commissionner' de New York puis Maire et enfin Président des Etats-Unis, qui multiplie les aveux d'impuissance : «No one could really police New York. ... He had no humor or real compassion or awareness of what could ever save the City", admet-il dans The Good Policeman. ${ }^{6}$ Dans Little Angel Street, le bilan est encore plus

35Jean Noël Blanc, Polarville - Images de la ville dans le roman policier, p.80 et p.83.

36Jerome Charyn, The Good Policeman, New York, The Mysterious Press, 199, p.106. 
amer, la chute inévitable : «Soon we'll all be homeless. The City is a cesspool. The plague is upon us, Isaac». ${ }^{37}$ Citizen Sidel clôt la série sur une note particulièrement noire: «Manhattan doesn't breed regular guys». ${ }^{38}$ La norme n'est pas de mise à New York: tout est poussé à l'extrême par la dureté de la ville. Le crime, toujours considéré comme un aspect marginal de la vie urbaine, devient ici la norme, une nécessité, un parcours obligé pour survivre dans un tel contexte. Tous y sont plongés, les riches par peur de voir leur statut menacé, les pauvres pour survivre ; le crime deviennent endémique, comme l'analyse Michael Woolf dans «Exploding the Genre»:

«The genre offers the potential for the development of an urban fiction which
has as its real concern the texture of contemporary experience, that uses crime
and criminality as metaphors for a reality in which social disorder, even evil, is
a perceived norm. Within that structure, it is also appropriate that the
detective should be a failed figure, precisely because his struggle is not so
much with the criminal but with a prevailing notion of reality. He achieves, at
best, a temporary illusion of control beneath which chaos prevails.... Isaac's
failure is assured precisely because he seeks to find answers to the
unanswerable. ... He both perceives the futile madness of the surrounding
world, and defies it, operating within a sense of universal psychosis and,
simultaneously, outside it, groping blindly toward some version of God's
grace. ... He is a "hero" because he both recognises his own impotence within
the prevailing psychosis, and struggles against it in massively futile
persistence. » ${ }^{39}$

L'impuissance reste un élément crucial du polar métaphysique: la ville se dresse toute-puissante face au héros qui ne la défie que parce qu'il ne peut accepter une telle situation de déchéance. Malgré tout, il ne possède pas le pouvoir suffisant pour inverser la tendance: il ne peut que rétablir la justice ça et là, de manière fragmentée, tout comme les indices qu'il collecte ne forment finalement jamais un tout qui conduit à la résolution de l'enquête. L'accès à une forme de rédemption, à une compréhension globale de la ville ne peut être atteint, le héros ne se bat que pour des fragments de vérité et de justice : la ville maintient son contrôle sur tout le reste. L'impuissance d'Isaac Sidel s'accompagne d'une ascension de l'échelle sociale jusqu'au poste le plus prestigieux : président des Etats-Unis. Au contraire des héros de The New York Trilogy il ne disparaît pas dans un abîme insondable et indéterminé, il ne chute pas. Cependant la ville l'affaiblit malgré tout par le paradoxe entre les

37Jerome Charyn, Little Angel Street, p.134.

${ }^{38}$ Jerome Charyn, Citizen Sidel, New York, Mysterious Press, 1999, p.18.

${ }^{39}$ Mike Woolf, «Exploding the Genre: The Crime Fiction of Jerome Charyn», in Brian Docherty (dir),

American Crime Fiction: Studies in the Genre, , New York, St. Martin's Press, 1988, p.132 and p.138. 
hautes fonctions qu'il occupe et le peu de pouvoir qu'il détient sur la ville. Cette impuissance le laisse tout comme les personnages de Paul Auster - Quinn, Blue et Fanshawe - marqué par d'irrémédiables séquelles psychiques et une solitude totale face à l'ampleur de sa tâche et son incapacité à l'exécuter. Jean-Noël Blanc emploie l'expression «ville-système» pour désigner une telle spirale urbaine d'où aucune évasion ne semble possible. ${ }^{40} \mathrm{Ce}$ terme semble en effet s'appliquer à la ville du polar métaphysique.

\section{Dystopie}

Si l'on considère Lionel Essrog et Conrad Metcalf chez Jonathan Lethem et Max Klein chez Paul Auster, leur principal point commun est d'avoir réussi à conclure leur enquête: la vérité éclate, le coupable est connu. Toutefois les détectives n'en retirent aucun bénéfice: leur succès s'accompagne d'un lourd tribut, celui de leur exclusion du système urbain. Evoluant jusqu'ici à sa marge, ils en sont désormais bannis sans pouvoir accéder à un éventuel ailleurs tant leur dépendance à la cité reste forte. Sans logement, argent ou profession, Metcalf replonge dans la drogue et se livre volontairement à une nouvelle peine de cryogénisation. Le répit procuré par le succès de son enquête est de courte durée:

«I drove the car up into the hills until I found a view I liked. Then I got out and looked at it. There was a wind coming up off the bay, and it brought with it a smell of salt. It made me think of the ocean, and I entertained a brief fantasy of taking the car and driving down the peninsula to find a beach where I could throw my make and the stuff that looked like money and maybe even my seventy-five points of karma into the surf and then stretch out on the sand and wait to see what happened. I played with it the way you can when you know you'll never do it.» (Lethem, 1994, 220-221) (11 $^{1}$

L'ailleurs, un instant présenté comme possible, s'évanouit définitivement, de même que l'idée de disparaître à la manière des détectives de The New York Trilogy. La nécessité de réintégrer le système urbain qu'il a toujours connu l'emporte, même si pour cela il doit accepter la place la plus marginale de ce cercle, celle de détenu. Dans Motherless Brooklyn, Essrog échappe lui aussi momentanément à New York quand son enquête le conduit dans un port du Maine. L'expérience hors de la ville le perturbe extrêmement, il ne peut la quitter sans se sentir totalement perdu :

«It was as I zipped and turned to see the ocean that the vertigo hit me. I'd found an edge, all right. Waves, sky, trees, Essrog - I was off the page now,

${ }^{40}$ Jean Noël Blanc, Polarville - Images de la ville dans le roman policier, p.112.

${ }^{41}$ Jonathan Letham, Gun, with Occasional Music, pp.220-21. 
away from the grammar of skyscrapers and pavement. I experienced it precisely as a loss of language, a great sucking-away of the word-laden walls that I needed around me, that I touched everywhere, leaned on for support, cribbed from when I ticced aloud. Those walls of language had always been in place, I understood now, audible to me until the sky of Maine deafened them with a shout of silence. I staggered, put one hand on the rocks to steady myself. I needed to reply in some new tongue, to find a way to assert a self that had become tenuous, shrunk to a shred of Brooklyn stumbling on the coastal void: Orphan meets ocean. $»^{42}$

Essrog réintègre lui aussi sa place dans le système new-yorkais en adoptant une profession tout à fait légale désormais. Il reste néanmoins en marge de la société de par son handicap et son absence de repères. Il compare d'ailleurs à plusieurs reprises New York à sa maladie, le syndrome de Tourette, pour en souligner la fatalité commune, l'absence de répit qu'elles engendrent avec cet exemple de l'addiction aux jeux de hasard:

«The docility was heart-breaking. The games were over almost before they started, the foil scraped off the tickets with a key or a dime, the contrived nearmisses underneath bared. (New York is a Tourettic city, and this great communal scratching and counting and tearing is a definite symptom.) The sidewalk just outside the Casino was strewn with discarded tickets, the chaff of wasted hope. $»^{43}$

S'évader de la dureté de la ville passe donc par de petites choses: l'espoir de gagner un peu d'argent par le jeu, ou bien le réconfort d'un repas, tel le «chicken shwarma, ... one of the great secret sandwiches of New York, redemption for a whole soulless airport». ${ }^{44}$ L'ironie est bien sûr présente dans ces pathétiques moments de répit pris sur la ville, car éphémères et ancrés dans la ville malgré tout. Dans «Access Fantasy», Jonathan Lethem présente aussi la ville comme système, un système d'où l'on ne s'échappe pas. La ville sépare nantis et démunis de chaque côté d'une frontière nommée «the One-Way Permeable Barrier».45 Les plus pauvres restent invisibles des plus riches. Une raison inconnue - l'implacabilité de la ville? - les a poussés à réunir quelques biens et prendre leur voiture pour fuir. Toutefois cette fuite n'a pu se concrétiser : la masse des véhicules se trouve bloquée dans un gigantesque embouteillage urbain dont le début et la fin restent indéfinis, rappelant une fois encore le rhizome. Il est impossible de quitter la ville, chacun attend le

${ }^{42 J}$ Jonathan Letham, Motherless Brooklyn, New York, Faber \& Faber, 2000, p.113.

43Jonathan Letham, Motherless Brooklyn, p.113.

${ }^{44}$ Jonathan Letham, Motherless Brooklyn, p.311.

45Jonathan Letham, «Access Fantasy», p.24. 
providentiel «start-up» qui débloquera la circulation. ${ }^{46}$ Or cet espoir s'amenuise à mesure que le temps passe:

«There was a start-up about a half mile ahead the day before, a fever of distant engines and horns honking as others signalled their excitement - a chance to move! - and so he'd spent the day jammed behind the wheel, living in his Apartment on Tape, waiting for that chance, listening under the drone of distant helicopters to hear the start-up make its way downtown. But the wave of revving engines stalled before reaching his street. He never even saw a car move, just heard them. Perhaps the start-up was only a panic begun by someone warming their motor, reviving their battery. ... Rumors had it Welfare Helicopters had been sighted ... and a lot of people had left their cars, drifted down that way, looking for easy cash. Which was one reason the startup died, it occurred to him - too many empty cars. Along with the cars that wouldn't start anymore.» ${ }^{47}$

La fuite hors de la ville qui semblait possible en prenant simplement sa voiture devient désormais tout à fait improbable. La ville tient malgré tout ses habitants prisonniers. C'est alors au tour de Max Klein de dresser le bilan d'une société régie par la loi urbaine: «People are wrong to say this is a preview of the end of civilization. It is the essence of civilization, the exact price we pay for being what we are and wanting what we want».48 L'ambition démesurée de l'homme, son désir de bâtir une cité à l'image de ses désirs les plus fous se voient punis par un retournement inattendu de pouvoir: la création prend le pas sur son créateur et inverse les rapports d'autorité dans une relecture du mythe du golem.

\section{La ville totale}

Ebranlé dans ses repères et forcé de retrouver sa simple condition d'homme soumis à des forces supérieures, l'individu doit affronter l'épreuve qui lui est imposée sans possibilité de s'en détacher, mais en conservant malgré tout l'espoir d'une éventuelle rédemption. Michael Woolf souligne d'ailleurs «the profoundest of paradoxes, the persistence of a notion of redemption in an ostensibly doomed and damned world» qui se traduit chez l'individu par «the heroic nature of flawed humanity as it crawls towards some bizarre version of spiritual salvation». ${ }^{49}$ Les termes de rédemption et de salut sont issus du champ lexical religieux mais revêtent plutôt dans le polar contemporain une valeur spirituelle. Dans ce cadre

"6onathan Letham, «Access Fantasy», p.23.

47Jonathan Letham, «Access Fantasy», p.24.

${ }^{48}$ Paul Auster (under the pseudonym of Paul Benjamin), Squeeze Play (1982), p.53.

${ }^{49}$ Mike Woolf, «Exploding the Genre: The Crime Fiction of Jerome Charyn», p.142. 
urbain étouffant, le détective ne fuit pas ; il n'y parvient pas car il est fait de la ville, elle fait partie de lui autant qu'il fait partie d'elle. Il y reste jusqu'à ce qu'elle ait usé toutes ses résistances physiques et mentales et qu'il ne soit plus en mesure de poursuivre la mission qu'il s'est fixée, souvent à contre-courant de la société qui l'entoure: ne pas se résigner face au crime, symbole même de la ville implacable. Dans le chapitre intitulé «Lutter, quand même», Jean-Noël Blanc soulève une question cruciale: «pourquoi se battre contre la ville?» et y suggère une réponse :

«La réponse est simple: par respect des valeurs humaines. La ville gagnera toujours, mais ou bien on abandonne, et alors c'est la mort de l'humain dans l'homme tout comme il y a le cadavre de l'urbanité dans l'urbain de ces villeslà, ou bien on lutte, au moins par souci de maintenir l'honneur. Par scrupule moral.»50

Le détective est en effet avant tout un homme. Si le polar traditionnel en avait fait un héros aux capacités et à l'hérö̈sme presque surhumains, le polar métaphysique le ramène à sa condition première, celle qui est la cause de ses échecs et de ses faiblesses - il n'est qu'un homme - mais qui motive aussi sa démarche de tenir bon et de conserver un sens moral. Cette lourde mission aux connotations là aussi teintées de religieux, ce que Blanc nomme «la robe de bure du missionnaire urbain» reste celle endossée par les détectives vulnérables de Paul Auster, Jonathan Lethem et Jerome Charyn, aux rangs desquels figure Isaac Sidel, qui déclare dans Citizen Sidel: «I wanna help New York» dans une tentative de réaffirmation du rôle de l'individu au sein de la ville. ${ }^{51}$

\footnotetext{
${ }^{50 J e a n ~ N o e ̈ l ~ B l a n c, ~ P o l a r v i l l e ~-~ I m a g e s ~ d e ~ l a ~ v i l l e ~ d a n s ~ l e ~ r o m a n ~ p o l i c i e r, ~ p .126 . ~}$

${ }^{51}$ ean Noël Blanc, Polarville, p.156.

Jerome Charyn, Citizen Sidel, p.104.
} 